IRSTI $41.21 .05 ; 41.21 .19$

https://doi.org/10.26577/phst.2021.v8.i2.07

\title{
Detecting the Sun's active region using image processing techniques
}

\author{
A. Sarsembayeva ${ }^{1, *}$ iD , M. Odsuren ${ }^{2, \uparrow}$ iD , F. Belisarova ${ }^{\text {iD , }}$ \\ A. Sarsembay ${ }^{3}$ iD and S. A. L. Maftunzada ${ }^{1,4}$ iD \\ ${ }^{1}$ Department of Physics and Technology, Al-Farabi Kazakh National University, Almaty, Kazakhstan \\ ${ }^{2}$ School of Engineering and Applied Sciences, National University of Mongolia, Ulaanbaatar, Mongolia \\ ${ }^{3}$ School-Lyceum №250 named after T.Komekbayev, Karmakchi, Kyzylorda, Kazakhstan \\ ${ }^{4}$ Faryab University, Kārte General Abdul Rashid Dostum Maymana, City Faryab Afghanistan \\ *e-mail: sarsembaeva.a@kaznu.kz; ’odsuren@seas.num.edu.mn
}

\begin{abstract}
The term 'space weather' refers to adverse conditions on the Sun that could affect the structures of space or terrestrial technology and risk human health or life. Flares produce effects that span the electromagnetic spectrum. During a flash, they emit x-rays and ultraviolet radiation, resulting in extremely high temperatures. The purpose of this work is to create a new algorithm for the identification of solar flares and active regions of the Sun, thus minimizing harm to spacecraft operations in orbit. An algorithm was developed for the automatic detection of active regions of the Sun (sunspots, solar flares), based on methods of image enhancement, segmentation, pattern recognition and mathematical morphology. The sun's surface also displays visible sunspots located in regions of the Sun that are magnetically active and whose number is an indication of the Sun's magnetic activity. In tracking and predicting solar activity, the identification and classification of sunspots are useful techniques. The main objective of this paper is to detect sunspots using images from the Solar Dynamics Observatory.
\end{abstract}

Key words: solar flares, sunspots, active region. PACS number(s): 96.60.-j; 96.60.Iv; 96.60.qe

\section{Introduction}

Sunspots are visible regions of the Sun's photosphere that reflect its activity. The automatic identification of sunspots from digital images is complicated by their shape and variable contrast irregularities and intensity compared to their surroundings. The detection of these sunspots and their characteristics, such as scale, contrast and location on the solar disk, plays an important role in the prediction of space weather and in the study of total solar irradiance, differential rotation, solar convection zone modeling [1], solar radius variability [2-8], and other significant phenomena. For irradiance studies, the area measurements of sunspots are important [9-12]. The measurement of sunspot areas has been shown to rely on the method of detection used and on the images themselves [13-14]. Then, inaccurate calculation of sunspot areas is the key barrier to irradiance modeling [15]. The presence of solar differential rotation is clearly demonstrated by the sunspots. It is easy to see that they are traveling in a few days from east to west on the solar disk. In order to locate sunspots and generate catalogues, many manual or automated methods have been used. Automatic methods use spatial filtering that also affects the resolution of the image and the properties of the sunspot [16-17]. To analyze a small number of images every day, manual methods are sufficient, but automated methods are required to classify these characteristics in recent years as a large and the amount of high-resolution solar images have been obtained from ground and space.The goal of this paper is to identify brightest area of a retinal image using naive and robust methods for the identification of sunspots in solar images.

The goal of this paper is to identify brightest area of a retinal image using naive and robust methods for the identification of sunspots in solar images.

Monitoring of solar flares in a real time is carried out by the Geostationary Operational Environmental Satellite or GOES [8]. Data on the electrons, protons, and X-rays were taken from satellites GOES 13, GOES 14 and GOES 15 [18-19]. 
On August 9, 2011, on the Sun, X class flares were registered. In total, 8 classes are distinguished in the scale of solar flares: A, B, C, M and X, each subsequent of which exceeds the previous power by 10-100 times. The event, more accurately estimated as X6.9, occurred in the morning and observed for about 20 minutes with a maximum at 08:05 UT [20].

On March 7, 2012 at 00:02 UT, another eruption of solar flare class X5.4 was registered. The event occurred in the active area of 11429 and was observed for about 22 minutes with a maximum at 00:24 UT. In total, 2 solar flares class of $\mathrm{X}$ were registered.

On November 5, 2013, around 22:07 on World time there was a fairly strong release of solar matter into space. The solar flare is estimated as X3.3 and its maximum was observed at 22:12 on world time. In total, 1 solar flare of class $\mathrm{X}$ were registered.

On February 25, 2014, 4 class C solar flares and 1 class $X$ solar flares were registered. In active area 11990 at 00:39 GMT, solar flare class of X4.9 occurred and was observed for about 10 minutes with a maximum at 00:49 UT.

On September 6, 2017 at 11:53, the X class flare $\mathrm{X} 9.3$ was registered. The event occurred in the active area of 12673 and was observed for about 9 minutes with a maximum at 12:02 UT. In total, 2 flares of class X, 3 flares of class $M$ and 2 flare of class $C$ were registered.

\begin{tabular}{|c|c|c|c|c|c|}
\hline Date & X-ray class & Active Region & Start time (hhmm) & End time (hhmm) & $\begin{array}{l}\text { Maximim time } \\
(\text { hhmm) }\end{array}$ \\
\hline 2011/08/09 & $\begin{array}{l}\text { M2.5 } \\
\text { C1.4 } \\
\mathbf{X 6 . 9} \\
\text { C2.2 } \\
\text { C2.4 } \\
\text { B8.6 } \\
\text { C2.0 } \\
\text { C3.9 }\end{array}$ & $\begin{array}{l}11263 \\
11263 \\
11263 \\
11263 \\
11263 \\
11263\end{array}$ & $\begin{array}{l}03: 19 \\
07: 19 \\
07: 48 \\
13: 29 \\
15: 43 \\
16: 58 \\
18: 04 \\
23: 33\end{array}$ & $\begin{array}{l}04: 08 \\
07: 27 \\
08: 08 \\
13: 57 \\
16: 04 \\
17: 05 \\
18: 42 \\
23: 49\end{array}$ & $\begin{array}{l}03: 54 \\
07: 23 \\
08: 05 \\
13: 45 \\
15: 54 \\
17: 02 \\
18: 11 \\
23: 43\end{array}$ \\
\hline $2012 / 03 / 07$ & $\begin{array}{l}\mathbf{X 5 . 4} \\
\text { X1.3 } \\
\text { C1.6 }\end{array}$ & $\begin{array}{l}11429 \\
11430 \\
11429\end{array}$ & $\begin{array}{l}00: 02 \\
01: 05 \\
16: 19\end{array}$ & $\begin{array}{l}00: 40 \\
01: 23 \\
16: 26\end{array}$ & $\begin{array}{l}00: 24 \\
01: 14 \\
16: 22\end{array}$ \\
\hline $2013 / 11 / 05$ & $\begin{array}{l}\text { C1.6 } \\
\text { M2.5 } \\
\text { C3.5 } \\
\text { C8.0 } \\
\text { C2.5 } \\
\text { C2.3 } \\
\text { C2.3 } \\
\text { C3.1 } \\
\text { M1.0 } \\
\text { C2.1 } \\
\text { C6.9 } \\
\mathbf{X 3 . 3}\end{array}$ & $\begin{array}{l}11889 \\
11890 \\
11885 \\
11890 \\
\\
11890 \\
11890 \\
11890 \\
11890\end{array}$ & $\begin{array}{c}05: 49 \\
08: 12 \\
09: 12 \\
11: 51 \\
13: 56 \\
16: 37 \\
16: 55 \\
17: 05 \\
18: 08 \\
19: 58 \\
21: 01 \\
22: 07\end{array}$ & $\begin{array}{l}06: 23 \\
08: 21 \\
09: 22 \\
12: 01 \\
14: 48 \\
16: 48 \\
17: 01 \\
17: 17 \\
18: 17 \\
20: 17 \\
21: 19 \\
22: 15\end{array}$ & $\begin{array}{c}06: 02 \\
08: 18 \\
09: 18 \\
11: 58 \\
14: 22 \\
16: 42 \\
16: 59 \\
17: 15 \\
18: 13 \\
20: 07 \\
21: 13 \\
22: 12\end{array}$ \\
\hline $2014 / 02 / 25$ & $\begin{array}{l}\mathbf{X} 4.9 \\
\text { C5.0 } \\
\text { C4.5 } \\
\text { C5.7 } \\
\text { C2.2 } \\
\end{array}$ & $\begin{array}{l}11990 \\
11989 \\
11986 \\
11986 \\
11984 \\
\end{array}$ & $\begin{array}{l}00: 39 \\
04: 27 \\
06: 16 \\
15: 04 \\
23: 31\end{array}$ & $\begin{array}{l}01: 03 \\
04: 36 \\
06: 28 \\
15: 18 \\
23: 37\end{array}$ & $\begin{array}{l}00: 49 \\
04: 32 \\
06: 20 \\
15: 10 \\
23: 34\end{array}$ \\
\hline 2017/09/06 & $\begin{array}{l}\mathrm{C} 1.6 \\
\mathrm{C} 2.7 \\
\mathrm{X} 2.2 \\
\mathbf{X 9 . 3} \\
\mathrm{M} 2.5 \\
\mathrm{M} 1.4 \\
\mathrm{M} 1.2\end{array}$ & $\begin{array}{l}12673 \\
12673 \\
12673 \\
12673 \\
12673 \\
12673\end{array}$ & $\begin{array}{l}06: 17 \\
07: 29 \\
08: 57 \\
11: 53 \\
15: 51 \\
19: 21 \\
23: 33\end{array}$ & $\begin{array}{c}06: 29 \\
07: 48 \\
09: 17 \\
12: 10 \\
16: 03 \\
19: 35 \\
23: 44\end{array}$ & $\begin{array}{l}06: 22 \\
07: 34 \\
09: 10 \\
12: 02 \\
15: 56 \\
19: 30 \\
23: 39\end{array}$ \\
\hline
\end{tabular}




\section{Determination of sunspots}

Template Matching is a way for a larger image to scan and locate the position of a template image. To this end, OpenCV comes with the cv2. matchTemplate() feature. It simply slides the image of the template over the input image (as in 2D convolution) and compares the template and the input image patch under the image of the template. Several comparison methods are implemented in OpenCV. This returns a grayscale image, where each pixel indicates how much of that pixel's neighborhood matches the template. In the Figure 1 (right panel) shown the brightest pixel location identification in AIA $335 \AA$ wavelengths obtained by using naive and robust methods.
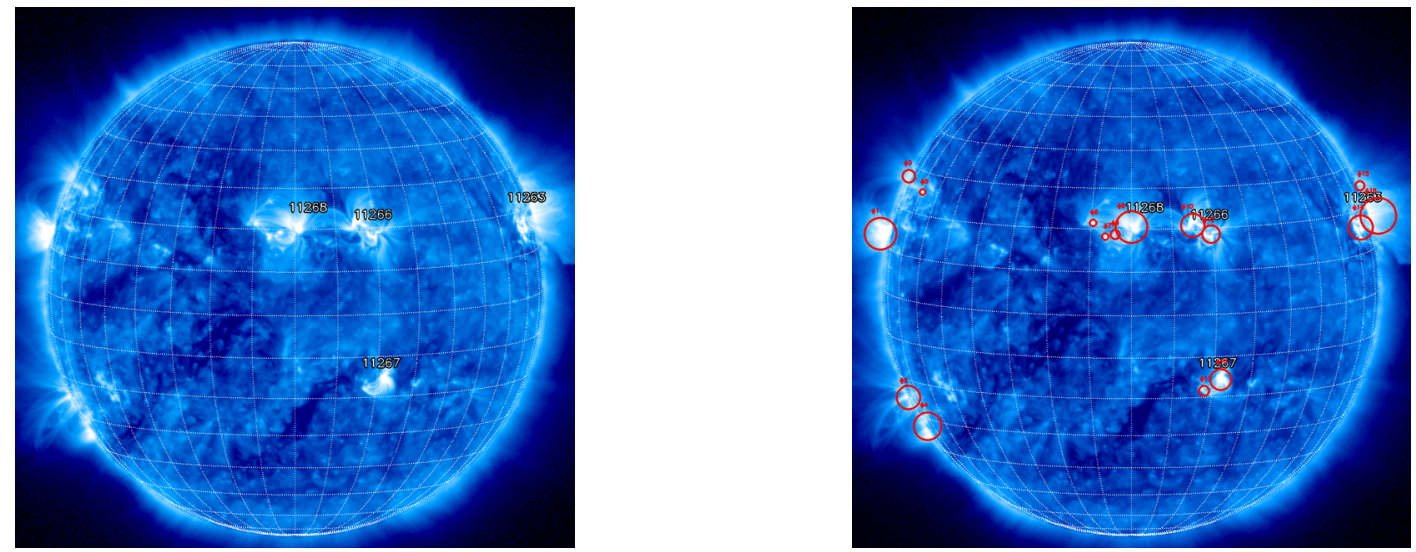

Figure 1 - Detecting the brightest area of a retinal image using naive and robust methods. (2011/08/09, X-ray class: X6.9, AR 1263 in AIA $335 \AA$ )

Using the cv2.minMaxLoc function without any pre-processing is the method of finding the brightest spot in an image. A single argument, which is our grayscale image, is needed for this purpose. This function then takes our grayscale image and, with the smallest and largest intensity values, finds the value of the pixel and (x,y) position, respectively. To break it down: minVal contains the smallest pixel intensity value, maxVal contains the largest pixel intensity value, minLoc specifies the $(\mathrm{x}, \mathrm{y})$ coordinates of minVal, and maxLoc specifies the (x, y) coordinates of maxLoc. In this application, we are only concerned with the pixel with the largest value, so we will grab that and draw a circle around the region and display it on. In the Figure 2 (right panel) shown the brightest pixel location identification in AIA $335 \AA$ wavelengths obtained by using naive and robust methods.

The cv2.minMaxLoc function without any pre-processing can leave extremely susceptible to noise. Instead, to eliminate high frequency noise, it is easier first to apply a Gaussian blur to the image. This way, their neighbors will balance out even pixels that have very high values (again, due to noise). From our command line statement, we apply our Gaussian blur with the radius supplied.
Then we will call cv2.minMaxLoc again to find the brightest pixel in the picture. Since we have applied a blurring pre-processing phase, however, we have averaged all pixels together with each other's supplied radius. This helps us to suppress high frequency noise and leaves cv2.minMaxLoc significantly less susceptible. We can average over a wider neighborhood of pixels by using a larger radius - thus mimicking larger regions of the image. Additionally, we can sum over smaller regions by using a smaller radius. In the Figure 3 (right panel) shown the brightest pixel location identification in AIA $335 \AA$ wavelengths obtained by using naive and robust methods.

Now, the naive cv2.minMaxLoc method finds this white pixel. The function is working correctly. It is indeed finding the single brightest pixel in the entire image. We are interested in the brightest region of the image, which is the optic nerve center. By utilizing a Gaussian blur, we are able to average a neighborhood of pixels within a given radius, and thus discard the single bright pixel and narrow in on the optic center region without an issue. In the Figure 4 (right panel) shown the brightest pixel location identification in AIA $335 \AA$ wavelengths obtained by using naive and robust methods. 

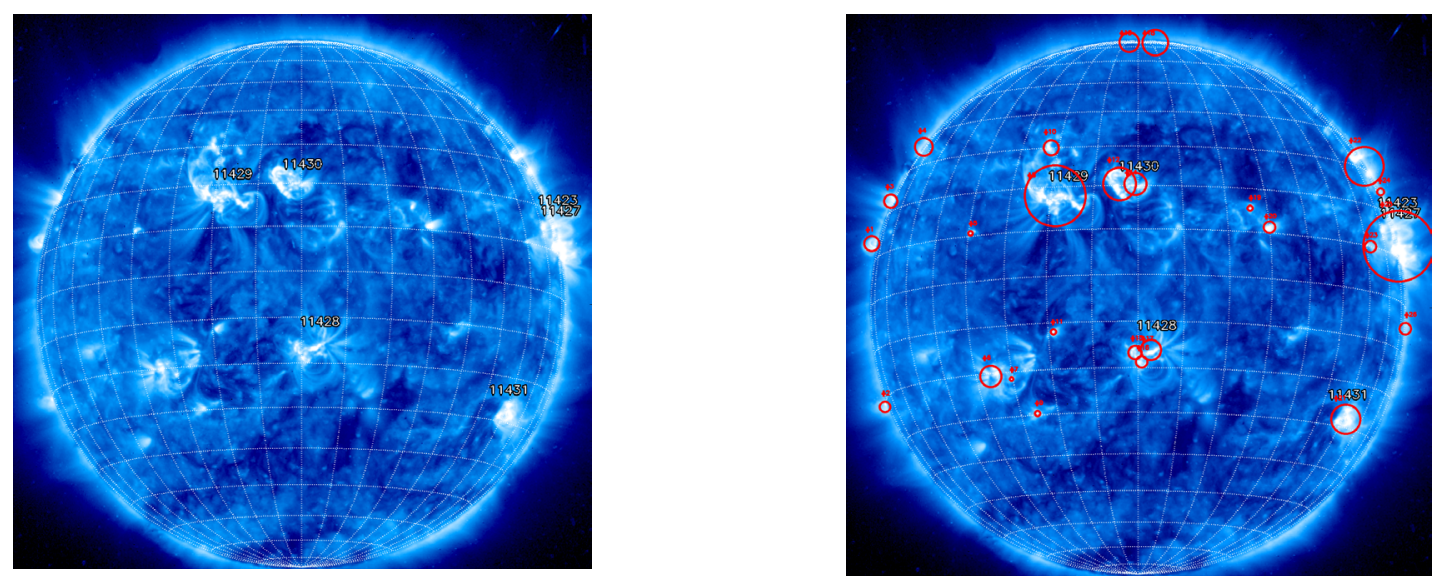

Figure 2 - Detecting the brightest area of a retinal image using naive and robust methods. (2012/03/07, X-ray class: X5.4, AR 1429 in AIA $335 \AA$ )
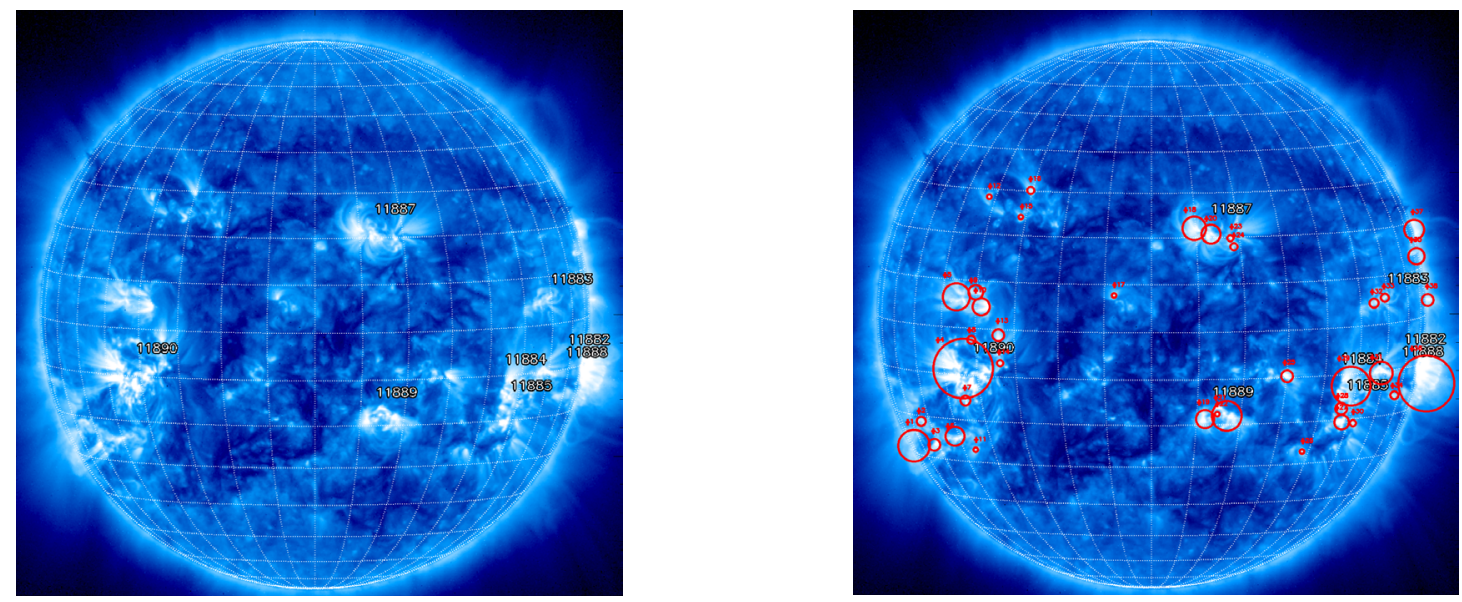

Figure 3 - Detecting the brightest area of a retinal image using naive and robust methods. (2013/11/05, X-ray class: X3.3, AR 1890 in AIA $335 \AA$ )
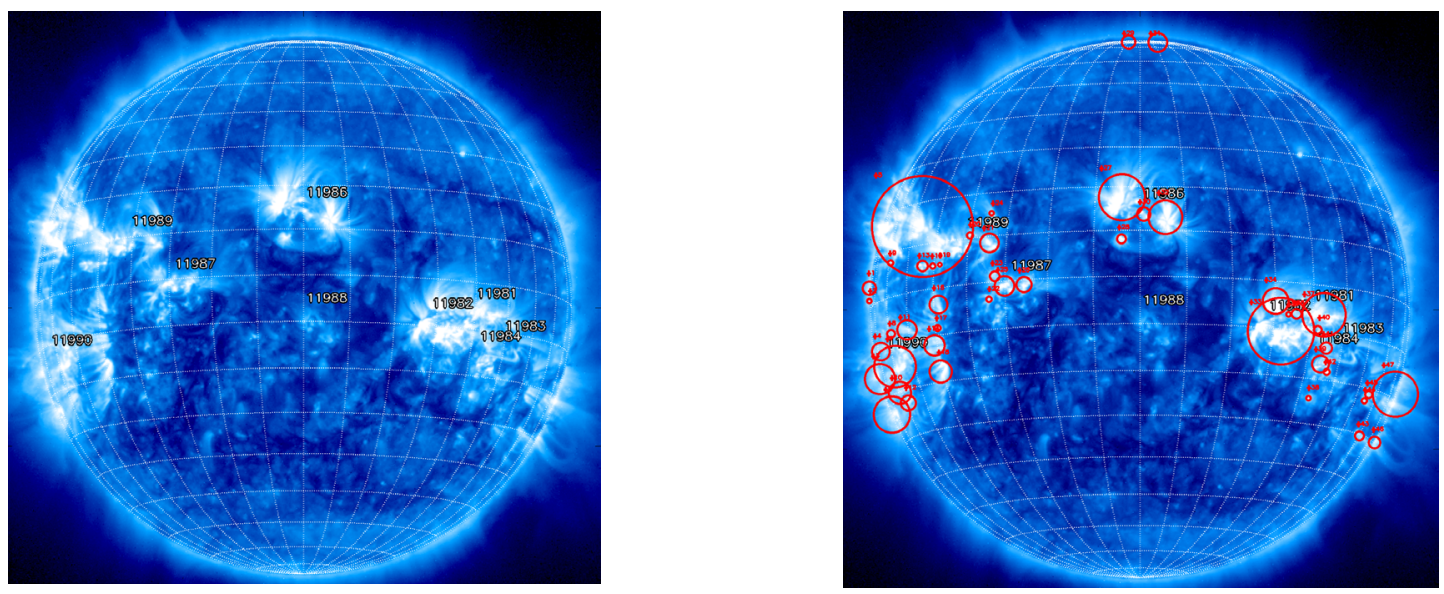

Figure 4 - Detecting the brightest area of a retinal image using naive and robust methods. (2014/02/25, X-ray class: X4.9, AR 1990 in AIA $335 \AA$ ) 
Obviously, a big aspect of getting the robust method to work correctly is properly setting your radius size. If the radius size is too small, we cannot find larger, brighter regions of the image. However, if we set the radius size too large, then we will detect too large of regions, missing out on the smaller ones, leading to sub-par results. Definitely spend some time playing with the radius size and viewing the results. In the Figure 5 (right panel) shown the brightest pixel location identification in AIA 335 $\AA$ wavelengths obtained by using naive and robust methods.
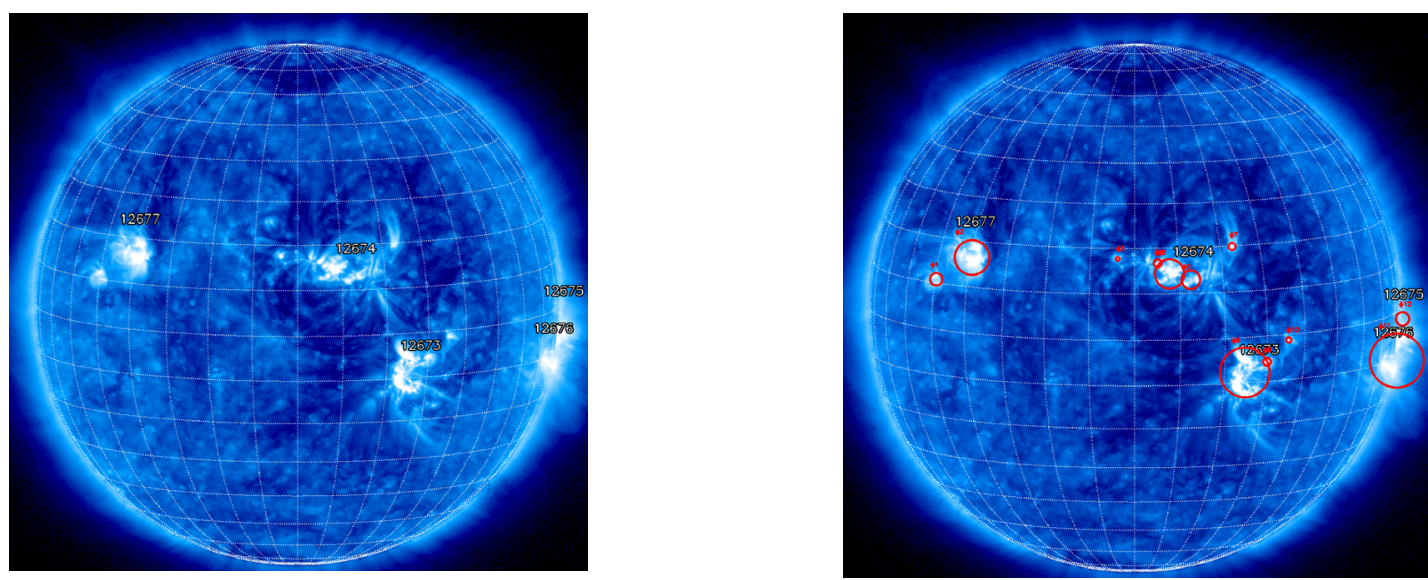

Figure 5 - Detecting the brightest area of a retinal image using naive and robust methods.

(2017/09/06, X-ray class: X9.3, AR 2673 in AIA $335 \AA$ )

\section{Conclusions}

In this work, we applied Gaussian blurring prior to finding the brightest spot in an image. By applying a Gaussian blur, we averaged the pixels within a given radius of each other together. Taking the average allows us to remove high frequency noise. We have used appropriate values for the radius of Gaussian blur. If we take too small of a value, we will mitigate the effects of the average and miss out on the larger, brighter regions. However, if the radius is too large, the small bright regions are not observable.

\section{Acknowledgments}

This work was partly supported by the Asian Research Center Foundation for Research program in National University of Mongolia.

\section{References}

1 S. Sofia, S. Basu, P. Demarque, L. Li, G. Thuillier. The nonhomologous nature of Solar diameter variations // Astrophys. J. Lett. - 2005. - V.632. - P. L147. https://doi.org/10.1086/498068

2 L. Laclare, C. Delmas, J.P. Coin, A. Irbah. Measurements and variations of the solar diameter // Solar Phys. 1996. - V.166. -P. 211. https://doi.org/10.1007/BF00149396

3 L. Győri, T. Baranyi, M. Turmon, J.M. Pap. SOHO 11 Symp. From Solar Min to Max: Half a Solar Cycle with SOHO // ESA SP-508. 2002. - P. 203.

4 T. Baranyi, L. Győri, A. Ludmány, H.E. Coffey. Comparison of sunspot area data bases // Mon. Not. Roy. Astron. Soc. - 2001. - V.323. - P.223. https://doi.org/10.1046/j.1365-8711.2001.04195.x

5 C. Fröhlich, J.M. Pap, H.S. Hudson. Improvement of the photometric sunspot index and changes of the diskintegrated sunspot contrast with time // Solar Phys. - 1994. - V.152. - P. 111. https://doi.org/10.1007/978-94-011-0950$5 \_18$

6 S. Zharkov, V. Zharkova, S. Ipson, A. Benkhalil. Technique for automated recognition of sunspots on full-disk solar images // EURASIP J. Appl. Signal Process. 15. - 2005. - P.2573. https://doi.org/10.1155/ASP.2005.2573

7 L. Győri, T. Baranyi, J. Murakozy, A. Ludmány. Comparison of sunspot area data determined from ground-based and space-borne observation // Mem. Soc. Astron. Ital. - 2005. V.76. - P. 985. 
8 ftp://ftp.ngdc.noaa.gov/STP/space-weather/solar-data/solar-features/solar-flares/x-rays/goes/

9 http://www.SolarMonitor.org

10 http://www.ngdc.noaa.gov/stp/satellite/goes/

11 A. Sarsembayeva, F. Belisarova, M. Odsuren, A. Sarsembay. February 25, 2014 solar flare data analysis in SunPy // Physical Sciences and Technology. - 2020. - Vol.7 (No. 3-4). - P. 22-26. https://doi.org/10.26577/phst.2020.v7.i2.03

12 M. J. Aschwanden, D. Alexander. Solar flare and CME observations with STEREO/EUVI // Solar Phys. - 2001. - V.204. - P.91. https://doi.org/10.1007/s11207-009-9347-4

13 K. J.H. Phillips, U. Feldman. Properties of cool flare with GOES class B5 to C2 // Astron. Astrophys. - 1995. V.304. - P.563.

14 H. A. Garcia. Forecasting methods for occurrence and magnitude of proton storms with solar hard X rays // Space Weather. - 2004. - V.2. - P.S06003. https://doi.org/10.1029/2003SW000035

15 Haisch, B., Strong, K.T., Rodono, M. Flares on the Sun and other Stars // Ann. Revs. Astron. Astrophys. - 1991. - V. 29. - P. 275.

16 A. T. Sarsembayeva, A. T. Sarsembay. Solar activity monitoring for the period April 10-20, 2017 // News of the National Academy of Sciences of the Republic of Kazakhstan-series Physico-Mathematical. - 2018. - V.2 (318). - P.9-11.

17 P.L. Bornmann. Limits to derived flare properties using estimates for the background fluxes-Examples from GOES // Astrophys. - 1990. - J.356. - P.733.

18 S.M. White, R.J. Thomas, R.A. Schwartz. Updated expressions for determining temperatures and emission measures from goes soft X-Ray measurements // Solar Physics. - 2005. V.227. - P.231. https://doi.org/10.1007/s11207-0052445-z

19 N. Gyenge, I. Ballai, T. Baranyi. Statistical study of spatio-temporal distribution of precursor solar flares associated with major flares // Monthly Notices of the Royal Astronomical Society. - 2016. - V. 459 (4). - P. 3532 - 3539. https:// doi.org/10.1093/mnras/stw859

20 Švestka Z., Cliver E.W. (1992) History and basic characteristics of eruptive flares. In: Švestka Z., Jackson B.V., Machado M.E. (eds) Eruptive Solar Flares. Lecture Notes in Physics, vol 399. Springer, Berlin, Heidelberg. https://doi. org/10.1007/3-540-55246-4_70 